\title{
Partially Adaptive Beamforming for Correlated Interference Rejection
}

\author{
Feng Qian, Student Member, IEEE, and Barry D. Van Veen, Member, IEEE
}

\begin{abstract}
Conventional linearly constrained adaptive beamformers often suffer from severe signal cancellation in the presence of interferers correlated with the signal. In this paper, we propose a partially adaptive beamforming technique for correlated interference rejection in broadband signal environments. The beamformer output mean squared error is decomposed into an interference mean squared error term and an additional signal cancellation term that is due to the presence of correlated interference. Both mean squared errors depend on the adaptation space. The partially adaptive beamforming technique proposed here chooses an adaptation space which results in little signal cancellation while maintaining satisfactory interference cancellation. It is shown that, for a given interference scenario, a partially adaptive beamformer can be designed such that maximum interference cancellation is achieved without any signal cancellation. In practice, an approximate design procedure is provided to accommodate a set of likely interference scenarios. Analysis of the feasibility of this approach is presented. The effectiveness of the technique is demonstrated through examples.
\end{abstract}

\section{INTRODUCTION}

$\mathbf{I}$ $T$ IS well-known that signal cancellation results when conventional linearly constrained adaptive beamformers are employed in environments containing interferers that are correlated with the signal [1]. In addition to cancelling uncorrelated interferers, the beamformer uses the correlated interferers to cancel part of the desired signal in order to achieve its goal of minimum output power. This often leaves the beamformer output virtually useless. Correlated interferers can occur due to multipath propagation or "smart jamming." Successful adaptive beamforming in correlated interference environments becomes, therefore, a very important issue.

Various methods for preventing signal cancellation have been proposed [2]-[4]. The predominant approach is use of averaging to destroy the correlation between signal and interference prior to beamforming. There are two primary means to achieve this: spatial averaging and frequency-domain averaging. The spatial averaging technique [2], [3] uses a bank of subarrays. The subarray data covariance matrices are averaged to reduce the correlation between the signal and interferers. Obviously, this technique only works for uniform array structures. If the correlated interferers originate from directions close to that of the desired signal, then large

\footnotetext{
Manuscript received November 11, 1993; revised June 13, 1994. This work was supported by the Aileen S. Andrew Foundation, the National Science Foundation under award MIP-8958559, and the Army Research Office under grant DAALO4-93-G-0208. The associate editor coordinating the review of this paper and approving it for publication was Prof. Daniel Fuhrmann.

The authors are with the Department of Electrical and Computer Engineering, University of Wisconsin, Madison, WI 53706 USA

IEEE Log Number 9407626
}

numbers of subarrays or long inter-subarray displacements are necessary in order to reduce the correlation to an acceptable level. Since the effective array aperture is only that of the subarray, the beamformer's interference cancellation capability is often severely compromised. In the case of broadband signals, correlation reduction may also be carried out via averaging in the frequency domain, the so-called coherent signal subspace (CSS) technique [4]. However, the CSS transformation preprocessor that implements frequency domain averaging is dependent on preliminary estimates of signal directions. Moreover, frequency domain averaging is only applicable to situations where the time delays between the desired signal and correlated interferers are greater than half of the reciprocal of the signal bandwidth. Both averaging techniques represent a two-stage procedure: a correlation reduction preprocessing step followed by conventional beamforming.

More recently, a split-polarity transformation (SPT) technique has been proposed [5]. The SPT processor reverses the phases of the interferers in the data using a priori information about the interference environment. Decorrelation is achieved by averaging the covariance matrices of the original and processed data. Since the SPT processor must maintain the original phase of the desired signal while reversing the phase of the interferers, the method is effective only if the desired signal and interferers are not closely located.

Here we propose a one-stage partially adaptive beamforming approach. The beamformer output mean squared error is defined and used to evaluate the performance of a beamformer in the presence of correlated interferers. Minimum mean squared error is shown to be equivalent to minimum output power in the absence of correlated interferers. In correlated interference environments, the mean squared error consists of the usual interference component and an additional signal cancellation component. Both terms are dependent on the choice of the adaptation space. Hence, we propose choosing the adaptive degrees of freedom subject to a constraint on the maximum signal cancellation over a set of likely interference scenarios. A constructive procedure for obtaining an adaptation space that satisfies the constraint is proposed. Note that this approach does not require a uniform array structure. Time and frequency domain analyses reveal the general conditions under which partially adaptive beamformers can successfully cancel interferers while preserving the signal. The analyses show that the partially adaptive beamforming approach is only effective with broadband signals. Simulations are provided to demonstrate the utility of the partially adaptive beamforming approach. Good performance is obtained with relatively small 
spatial separations and short time delays between the signal and correlated interferers.

The paper is organized as follows. Section II introduces the class of linearly constrained minimum variance beamformers and the concept of partially adaptive beamforming. The mean squared error criterion for evaluating adaptive beamformer performance in correlated interference environments is established in Section III. In Section IV, the partially adaptive beamforming solution is developed. An analysis of this approach is provided in Section V. Examples illustrating the effectiveness of partially adaptive beamforming approach are furnished in Section VI, and a summary is given in Section VII. Throughout the paper, lower and upper case boldface symbols represent vectors and matrices respectively. Superscript $H$ denotes complex conjugate transpose.

\section{ADAPTIVE BEAMFORMING}

\section{A. Linearly Constrained Minimum Variance Beamforming}

Let the $n$-dimensional vector $x$ represent the data received at the sensor outputs (and delayed versions of the sensor outputs if FIR filters are employed). The beamformer output $y$ is an inner product of the beamformer weight vector $w$ and the data vector $x$

$$
y=w^{H} \boldsymbol{x}
$$

The linearly constrained minimum variance (LCMV) criterion [6] for choosing $\boldsymbol{w}$ is

$$
\min _{\boldsymbol{w}} \boldsymbol{w}^{H} \boldsymbol{R}_{x} \boldsymbol{w} \quad \text { subject to } C^{H} \boldsymbol{w}=\boldsymbol{g}
$$

where $\boldsymbol{R}_{x}=E\left\{\boldsymbol{x} x^{H}\right\}, \boldsymbol{C}$ is the $n \times m$ constraint matrix, and $g$ is the $m$ dimensional response vector. Minimization of output power reduces the contributions of interference and noise to the beamformer output while the $m$ linear constraints are employed to prevent distortion of the desired signal. A generalized sidelobe canceller (GSC) representation [7] for the LCMV weight vector is

$$
\boldsymbol{w}=\boldsymbol{w}_{o}-C_{n} \boldsymbol{w}_{a} .
$$

The GSC structure is illustrated in Fig. 1. Here, $\boldsymbol{w}_{o}$ is a nonadaptive weight vector that satisfies the constraints $C^{H} \boldsymbol{w}_{o}=$ g. The $n \times q(q=n-m)$ full rank matrix $\boldsymbol{C}_{n}$ satisfies $C^{H} C_{n}=\boldsymbol{O}$. Therefore, the $q$ dimensional adaptive weight $\boldsymbol{w}_{a}$ is unconstrained and the minimization problem (2) becomes

$$
\min _{\boldsymbol{w}_{\mathrm{a}}}\left(\boldsymbol{w}_{o}-\boldsymbol{C}_{n} \boldsymbol{w}_{a}\right)^{H} \boldsymbol{R}_{x}\left(\boldsymbol{w}_{o}-\boldsymbol{C}_{n} \boldsymbol{w}_{a}\right) .
$$

The solution to (4) is obtained as

$$
\boldsymbol{w}_{a}=\left(\boldsymbol{C}_{n}^{H} \boldsymbol{R}_{x} \boldsymbol{C}_{n}\right)^{-1} \boldsymbol{C}_{n}^{H} \boldsymbol{R}_{x} \boldsymbol{w}_{o} .
$$

$C_{n}$ is termed the signal blocking matrix. ${ }^{1}$ We refer to range $\left(\boldsymbol{C}_{n}\right)$ as the adaptation space because $\boldsymbol{w}$ can only adapt the components that lie in this space.

\footnotetext{
${ }^{1}$ The portion of $x(k)$ due to the signal generally lies in the space spanned by the columns of $C$
}

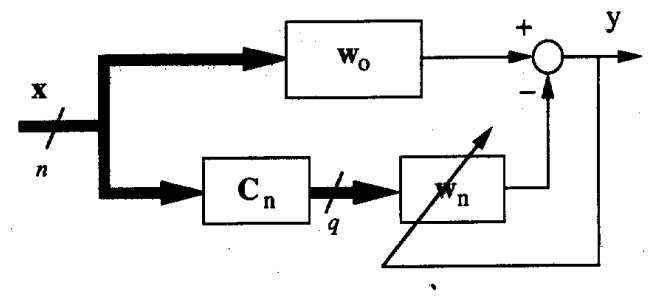

Fig. 1. The GSC beamformer implementation.

\section{B. Partially Adaptive Beamforming}

In a partially adaptive beamformer, the original adaptation space $\operatorname{range}\left(\boldsymbol{C}_{n}\right)$ is mapped into a subspace through a fixed $q \times p(p<q)$ full-rank transformation matrix $\boldsymbol{T}$ to yield a lower dimensional adaptation space $\operatorname{range}\left(\boldsymbol{T}_{n}\right)$ where

$$
T_{n}=C_{n} T \text {. }
$$

A pictorial illustration of the GSC structure for a partially adaptive beamformer is obtained from Fig. 1 by replacing $C_{n}$ by $\boldsymbol{T}_{n}$. The corresponding optimal adaptive weight vector is

$$
\boldsymbol{w}_{a}=\left(\boldsymbol{T}_{n}^{H} \boldsymbol{R}_{x} \boldsymbol{T}_{n}\right)^{-1} \boldsymbol{T}_{n}^{H} \boldsymbol{R}_{x} \boldsymbol{w}_{o}
$$

In uncorrelated interference environments, partially adaptive beamformers are employed to reduce the computational complexity and improve the convergence rate of adaptive algorithms involving large number of sensor arrays [6], [8], [9].

\section{BEAMFORMER PERFORMANCE CRITERION}

In this section, the beamformer mean squared error is defined. This mean squared error is then used to evaluate the performance of an adaptive beamformer with adaptation space range $\left(\boldsymbol{T}_{n}\right)$ in the presence of correlated interferers.

\section{A. Beamformer Mean Squared Error}

Let $s$ and $\boldsymbol{n}$ be the portions of $\boldsymbol{x}$ due to the desired signal and interference respectively, i.e.

$$
\boldsymbol{x}=\boldsymbol{s}+\boldsymbol{n}
$$

then

$$
\boldsymbol{R}_{x}=\boldsymbol{R}_{s}+\boldsymbol{R}_{s n}+\boldsymbol{R}_{n s}+\boldsymbol{R}_{n}
$$

where

$$
\begin{aligned}
\boldsymbol{R}_{\boldsymbol{s}} & =E\left\{\boldsymbol{s s ^ { H }}\right\} \\
\boldsymbol{R}_{n} & =E\left\{\boldsymbol{n} \boldsymbol{n}^{H}\right\} \\
\boldsymbol{R}_{\boldsymbol{s} n} & =\boldsymbol{R}_{n \boldsymbol{s}}^{H}=E\left\{\boldsymbol{s} \boldsymbol{n}^{H}\right\} .
\end{aligned}
$$

The terms $\boldsymbol{R}_{s n}$ and $\boldsymbol{R}_{n s}$ represent the correlation between the desired signal and the interference.

The desired beamformer output is

$$
s=\boldsymbol{w}_{o}^{H} s=\boldsymbol{w}^{H} s .
$$

Hence, the desired output signal power is

$$
P_{s}=w_{o}^{H} \boldsymbol{R}_{s} w_{o}
$$


The beamformer mean squared error $M S E$ is defined as the mean squared distance between the desired signal and the beamformer output, i.e.

$$
M S E=E\left\{\left|s-\boldsymbol{w}^{H} \boldsymbol{x}\right|^{2}\right\}
$$

An effective beamformer should exhibit small MSE. Substituting (10) and (8) into (12) results in

$$
M S E=E\left\{\left|\boldsymbol{w}^{H} \boldsymbol{n}\right|^{2}\right\}=\boldsymbol{w}^{H} \boldsymbol{R}_{n} \boldsymbol{w} .
$$

\section{B. MSE Decomposition for Optimal Weight Vector}

In order to see the impact of correlated interference on beamformer performance, the $M S E$ associated with the optimal beamformer weight vector (7) is decomposed into two terms: the term due to the interference and an additional term due to the presence of correlation between the signal and interference.

Since the constraint matrix $C$ is designed to provide specified response to the desired signal, $\boldsymbol{s} \in \operatorname{range}(\boldsymbol{C})=$ range $^{\perp}\left(\boldsymbol{C}_{n}\right) \subset$ range $^{\perp}\left(\boldsymbol{T}_{n}\right)$, and consequently

$$
T_{n}^{H} R_{s n}=O \text {. }
$$

Subsititution of (9) into (7) yields

$$
\boldsymbol{w}_{a}=\left(\boldsymbol{T}_{n}^{H} \boldsymbol{R}_{n} \boldsymbol{T}_{n}\right)^{-1} \boldsymbol{T}_{n}^{H}\left(\boldsymbol{R}_{n}+\boldsymbol{R}_{n s}\right) \boldsymbol{w}_{o}
$$

Note that the weight vector in (14) is decomposed into two components as

$$
\boldsymbol{w}_{a}=\boldsymbol{w}_{n}+\boldsymbol{w}_{s}
$$

where

$$
\begin{aligned}
& \boldsymbol{w}_{n}=\left(\boldsymbol{T}_{n}^{H} \boldsymbol{R}_{n} \boldsymbol{T}_{n}\right)^{-1} \boldsymbol{T}_{n}^{H} \boldsymbol{R}_{n} \boldsymbol{w}_{o} \\
& \boldsymbol{w}_{s}=\left(\boldsymbol{T}_{n}^{H} \boldsymbol{R}_{n} \boldsymbol{T}_{n}\right)^{-1} \boldsymbol{T}_{n}^{H} \boldsymbol{R}_{n s} \boldsymbol{w}_{o} .
\end{aligned}
$$

For a given adaptation space, $w_{n}$ is the optimal adaptive weight vector obtained in the absence of correlated interference. It is considered the "best" adaptive weight vector available in the sense that it provides the maximum interference cancellation without causing any signal cancellation. The term $w_{s}$ is due to the presence of correlated interference. It is responsible for the signal cancellation.

As illustrated in Fig. 2, the signal and interference components at the output of the nonadaptive beamformer $\boldsymbol{w}_{o}$ are $s=\boldsymbol{w}_{o}^{H} s$ and $n_{o}=\boldsymbol{w}_{o}^{H} \boldsymbol{n}$, respectively. The output of the adaptive branch consists of $n_{n}=\left(\boldsymbol{T}_{n} \boldsymbol{w}_{n}\right)^{H} \boldsymbol{n}$ and $n_{s}=\left(\boldsymbol{T}_{n} \boldsymbol{w}_{s}\right)^{H} \boldsymbol{n}$. Define

$$
n_{i}=n_{o}-n_{n}=\left(\boldsymbol{w}_{o}-\boldsymbol{T}_{n} \boldsymbol{w}_{n}\right)^{\boldsymbol{H}} \boldsymbol{n} .
$$

In the absence of correlated interference, $n_{i}$ is the interference portion of the beamformer output. The term $n_{s}$ is the additional component due to the presence of correlated interference; it is primarily used to cancel the desired signal. One can easily verify that

$$
E\left\{n_{i}^{*} n_{s}\right\}=0
$$

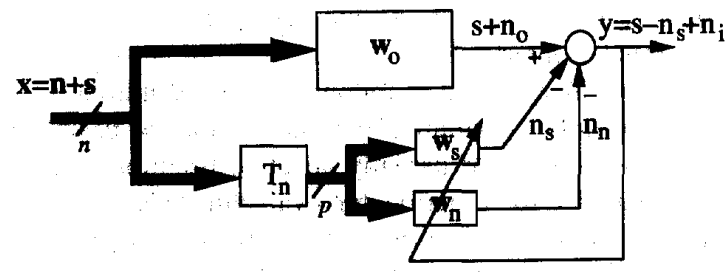

Fig. 2. Decomposition of signals in GSC structure.

Therefore, the $M S E$ associated with the optimal adaptive weight (14) is

$$
\begin{aligned}
M S E & =E\left\{\left|n_{i}-n_{s}\right|^{2}\right\}=E_{i}+E_{s} \\
E_{i} & =E\left\{\left|n_{i}\right|^{2}\right\} \\
E_{s} & =E\left\{\left|n_{s}\right|^{2}\right\} .
\end{aligned}
$$

$E_{i}$ and $E_{s}$ are respectively termed the interference $M S E$ and the signal cancellation $M S E .^{2}$ Let

$$
\begin{aligned}
P_{n o}= & E\left\{\left|n_{o}\right|^{2}\right\}=\boldsymbol{w}_{o}^{H} \boldsymbol{R}_{n} \boldsymbol{w}_{o} \\
P_{n n}\left(\boldsymbol{T}_{n}\right)= & E\left\{\left|n_{n}\right|^{2}\right\}=\boldsymbol{w}_{o}^{H} \boldsymbol{R}_{n} \boldsymbol{T}_{n}\left(\boldsymbol{T}_{n}^{H} \boldsymbol{R}_{n} \boldsymbol{T}_{n}\right)^{-1} \\
& \cdot \boldsymbol{T}_{n}^{H} \boldsymbol{R}_{n} \boldsymbol{w}_{o} \\
P_{s c}\left(\boldsymbol{T}_{n}\right)= & \boldsymbol{w}_{o}^{H} \boldsymbol{R}_{s n} \boldsymbol{T}_{n}\left(\boldsymbol{T}_{n}^{H} \boldsymbol{R}_{n} \boldsymbol{T}_{n}\right)^{-1} \boldsymbol{T}_{n}^{H} \boldsymbol{R}_{n s} \boldsymbol{w}_{o}
\end{aligned}
$$

It can be shown easily that

$$
\begin{aligned}
& E_{i}=P_{n o}-P_{n n}\left(T_{n}\right) \\
& E_{s}=P_{s c}\left(T_{n}\right) .
\end{aligned}
$$

Note that $P_{n o}$ is the interference power at the nonadaptive branch output. $P_{n n}\left(T_{n}\right)$ is the interference power at the adaptive branch output in the absence of correlated interference; it represents the beamformer's interference cancellation capability. $P_{s c}\left(T_{n}\right)$ represents the beamformer's signal cancellation capability in the presence of correlated interference; it is equal to the signal cancellation $M S E$.

In uncorrelated interference environments $R_{s n}=O$, hence $P_{s c}\left(\boldsymbol{T}_{n}\right)=0$, and the beamformer output power is

$$
P_{o}=\boldsymbol{w}_{o}^{H} \boldsymbol{R}_{s} \boldsymbol{w}_{o}+\boldsymbol{w}^{H} \boldsymbol{R}_{n} \boldsymbol{w}=P_{s}+M S E .
$$

The minimum output power is $P_{o}^{\text {min }}=P_{s}+E_{i}$. Therefore, minimum $M S E=E_{i}$ is achieved via output power minimization. However, in correlated interference environments

$$
P_{o}=P_{s}+M S E+w_{o}^{H} R_{s n} w+w^{H} \boldsymbol{R}_{n s} w_{o} .
$$

The minimum output power is now

$$
\begin{aligned}
P_{o}^{\min }= & P_{s}+P_{n o}-P_{n n}\left(\boldsymbol{T}_{n}\right) \\
& -P_{s c}\left(\boldsymbol{T}_{n}\right)+\boldsymbol{w}_{o}^{H} \boldsymbol{R}_{s n} \boldsymbol{w}_{o}+\boldsymbol{w}_{o}^{H} \boldsymbol{R}_{n s} \boldsymbol{w}_{o} \\
& -P_{n s}\left(\boldsymbol{T}_{n}\right)-P_{n s}^{*}\left(\boldsymbol{T}_{n}\right)
\end{aligned}
$$

where

$$
P_{n s}\left(\boldsymbol{T}_{n}\right)=\boldsymbol{w}_{o}^{H} \boldsymbol{R}_{n} \boldsymbol{T}_{n}\left(\boldsymbol{T}_{n}^{H} \boldsymbol{R}_{n} \boldsymbol{T}_{n}\right)^{-1} \boldsymbol{T}_{n}^{H} \boldsymbol{R}_{n s} \boldsymbol{w}_{o} .
$$

${ }^{2} E_{s}$ actually represents both the signal cancellation and changes in interference cancellation that occur as a result of correlated interference between the signal and interference. However, the signal cancellation is usually the dominant and most significant effect. Hence, we refer to $E_{s}$ as the signal cancellation $M S E$. 
Due to the presence of the cross terms, both signal and interference are suppressed when minimum output power is achieved. Hence, output power minimization does not yield small $M S E$ in the presence of correlated interference.

\section{Partially Adaptive Beamforming Solution}

The dependence of signal and interference cancellation on the adaptation space is demonstrated in the previous section. Here we propose restricting the degrees of freedom in the beamformer to limit signal cancellation capability.

\section{A. Motivation}

It is straightforward to show that

$$
\begin{aligned}
P_{n n}\left(\boldsymbol{T}_{n}\right) & \leq P_{n n}\left(\boldsymbol{C}_{n}\right) \\
P_{s c}\left(\boldsymbol{T}_{n}\right) & \leq P_{s c}\left(\boldsymbol{C}_{n}\right)
\end{aligned}
$$

if range $\left(\boldsymbol{T}_{n}\right) \subset \operatorname{range}\left(\boldsymbol{C}_{n}\right)$. That is, both signal cancellation capability and interference cancellation capability degrade when the dimension of the adaptation space is reduced. The goal is to choose the adaptation space so that the signal cancellation capability is greatly reduced while any loss in interference cancellation capability is minimized. If this goal is achieved, then the output power minimization criterion will yield a small $M S E$.

It is easy to show that

$$
\begin{aligned}
P_{n n}\left(\boldsymbol{T}_{n}\right) & =z_{n}^{H} \boldsymbol{P}\left(\boldsymbol{T}_{n}\right) z_{n} \\
P_{s c}\left(\boldsymbol{T}_{n}\right) & =z_{s}^{H} \boldsymbol{P}\left(\boldsymbol{T}_{n}\right) z_{s}
\end{aligned}
$$

where

$$
\begin{aligned}
& \boldsymbol{P}\left(\boldsymbol{T}_{n}\right)= \boldsymbol{R}_{n}^{H / 2} \boldsymbol{T}_{n}\left(\boldsymbol{T}_{n}^{H} \boldsymbol{R}_{n} \boldsymbol{T}_{n}\right)^{-1} \boldsymbol{T}_{n}^{H} \boldsymbol{R}_{n}^{1 / 2} \\
& \text { is the projection matrix onto } \quad \operatorname{range}\left(\boldsymbol{R}_{n}^{H / 2} \boldsymbol{T}_{n}\right) \\
& \boldsymbol{z}_{n}=\boldsymbol{P}\left(\boldsymbol{C}_{n}\right) \boldsymbol{R}_{n}^{H / 2} \boldsymbol{w}_{o} \\
& \boldsymbol{z}_{s}=\boldsymbol{P}\left(C_{n}\right) \boldsymbol{R}_{n}^{-(1 / 2)} \boldsymbol{R}_{n s} \boldsymbol{w}_{o} .
\end{aligned}
$$

Note that $\boldsymbol{P}\left(\boldsymbol{T}_{n}\right) \boldsymbol{P}\left(\boldsymbol{C}_{n}\right)=\boldsymbol{P}\left(\boldsymbol{T}_{n}\right)$ since $\operatorname{range}\left(\boldsymbol{P}\left(\boldsymbol{C}_{n}\right)\right) \subset$ range $\left(\boldsymbol{P}\left(\boldsymbol{T}_{n}\right)\right)$. The goal of partially adaptive beamformer design is to choose $T_{n}$ such that $P_{s c}\left(T_{n}\right) \approx 0$ and $P_{n n}\left(T_{n}\right) \approx$ $P_{n n}\left(C_{n}\right)$.

There is no signal cancellation if $P\left(T_{n}\right) z_{s}=o$. This is achieved if

$$
\boldsymbol{T}_{n}^{H} \boldsymbol{R}_{n s} \boldsymbol{w}_{\mathrm{o}}=\boldsymbol{o} .
$$

Recall $\boldsymbol{T}_{n}=\boldsymbol{C}_{n} \boldsymbol{T}$, so (39) implies $\boldsymbol{T}^{H} \boldsymbol{C}_{n}^{H} \boldsymbol{R}_{n s} \boldsymbol{w}_{o}=\boldsymbol{o}$. Such a $T$ always exists provided $p \leq q-1$. Thus, only one adaptive degree of freedom must be removed from the fully adaptive beamformer to prevent signal cancellation.

Removal of this degree of freedom will generally decrease the beamformer's interference cancellation. However, while this degree of freedom must be excluded from the adaptive portion of the GSC to prevent signal cancellation, it can be included in the nonadaptive portion to enhance interference cancellation, as suggested in [10]. Let $U_{n}$ be the unused adaptation space, i.e.

$$
\operatorname{range}\left(\boldsymbol{T}_{n}\right) \oplus \operatorname{range}\left(\boldsymbol{U}_{n}\right)=\operatorname{range}\left(\boldsymbol{C}_{n}\right)
$$

where $\oplus$ denotes the direct sum of subspaces. If the partially adaptive beamformer has nonadaptive weight vector

$$
\boldsymbol{w}_{q}=\boldsymbol{w}_{o}-\boldsymbol{U}_{n}\left(\boldsymbol{U}_{n}^{H} \boldsymbol{R}_{m} \boldsymbol{U}_{n}\right)^{-1} \boldsymbol{U}_{n} \boldsymbol{R}_{m} \boldsymbol{w}_{o}
$$

where

$$
\boldsymbol{R}_{m}=\boldsymbol{R}_{n}^{1 / 2}\left(\boldsymbol{I}-\boldsymbol{P}\left(\boldsymbol{T}_{n}\right)\right) \boldsymbol{R}_{n}^{H / 2}
$$

then it can be shown that $P_{n n}\left(\boldsymbol{w}_{q}, \boldsymbol{T}_{n}\right)=P_{n n}\left(\boldsymbol{w}_{o}, \boldsymbol{C}_{n}\right)$, while $P_{s c}\left(\boldsymbol{w}_{q}, \boldsymbol{T}_{n}\right)=P_{s c}\left(\boldsymbol{w}_{o}, \boldsymbol{T}_{n}\right)$. Hence, when the nonadaptive weight vector is chosen according to (41), fully adaptive interference cancellation is obtained with partially adaptive signal cancellation.

\section{B. Partially Adaptive Beamformer Design}

The above discussion assumes the interference scenario is known; in practice it is generally unknown. Here we use a parameterized interference model to accomodate uncertainty in the interference scenario. Let the vector $\boldsymbol{\theta}$ parameterize the interference environment. For example, $\boldsymbol{\theta}$ may represent the number of interferers, their locations, spectral characteristics, etc. $\boldsymbol{R}_{n}$ and $\boldsymbol{R}_{n s}$ are assumed to be completely determined by $\boldsymbol{\theta}$, and their explicit dependence on $\boldsymbol{\theta}$ is indicated by the notation $\boldsymbol{R}_{n}(\boldsymbol{\theta})$ and $\boldsymbol{R}_{n s}(\boldsymbol{\theta})$. The set of interference scenarios over which $\boldsymbol{T}_{n}$ is designed is represented by a discrete set $\mathcal{Q}=\left\{\boldsymbol{\theta}_{k}, k=1,2, \cdots, K\right\}$. Here $K$ is chosen so that the $\boldsymbol{\theta}_{k}$ adequately sample the range of interference scenarios of interest.

An average mean squared error minimization partially adaptive beamformer design procedure was proposed in [11]. However, this procedure results in a very complicated optimization problem. An alternate solution is pursued here by limiting the beamformer's signal cancellation for each scenario within the set $\mathcal{Q}$. That is, we choose $\boldsymbol{T}_{n}$ to satisfy

$$
P_{s c}\left(\boldsymbol{T}_{n}, \boldsymbol{\theta}\right) \leq \delta_{o}, \forall \boldsymbol{\Theta} \in \mathcal{Q}
$$

for some positive $\delta_{o}$. Note that $\delta_{o}$ is the upper bound on signal cancellation for any interference scenario in $\mathcal{Q}$. It may be chosen as a small fraction of the expected signal output power. Satisfaction of (43) generally requires removal of multiple degrees of freedom from the fully adaptive beamformer adaptation space.

The procedure proposed here for choosing a $T_{n}$ that satisfies (43) is similar in spirit to the point design procedure described in [12] for partially adaptive beamformer design in noncorrelated interference environments. The strategy is to remove from the original basis of $\operatorname{range}\left(\boldsymbol{C}_{n}\right)$ those basis vectors that lead to severe signal cancellation. At an interference scenario $\boldsymbol{\theta}$ of interest, $P_{s c}\left(\boldsymbol{T}_{n}, \boldsymbol{\theta}\right)$ is computed using the current adaptation space range $\left(\boldsymbol{T}_{n}\right)$. If $P_{s c}\left(\boldsymbol{T}_{n}, \boldsymbol{\theta}\right)$ exceeds the specified tolerance, the adaptation space is reduced by one dimension, i.e., a column in $\operatorname{range}\left(\boldsymbol{T}_{n}\right)$ is extracted. This process is carried out in a sequential order through all possible scenarios. Since $P_{s c}\left(\boldsymbol{T}_{n}, \boldsymbol{\theta}\right)$ only decreases as the adaptation space dimension is reduced, removal of additional columns never enhances the beamformer's signal cancellation capability. 
In order to achieve condition (39) at a given interference scenario $\boldsymbol{\theta}$, we propose removing $\boldsymbol{t}_{\boldsymbol{s}}(\boldsymbol{\theta})=\boldsymbol{T}_{n} \boldsymbol{T}_{n}^{H} \boldsymbol{R}_{n s}(\boldsymbol{\theta}) \boldsymbol{w}_{o}$ from $\boldsymbol{T}_{n}$. Many methods can be used to remove a given basis vector from a subspace spanned by a set of basis vectors. We adopt the following $\mathrm{QR}$ decomposition technique. Let

$$
\left[\begin{array}{ll}
t_{s}(\theta) & T_{n}
\end{array}\right]=Q R=\left[\begin{array}{lll}
q & T_{n}^{\prime} & V_{n}
\end{array}\right] R
$$

where $\boldsymbol{Q}$ is a unitary matrix and $\boldsymbol{R}$ is an upper triangular matrix. Since $R$ is upper triangular and $t_{s}(\Theta) \neq o$, the first column of $Q, q$, represents the same 1-D space as $t_{s} . T_{n}^{\prime}$ contains the columns of $\boldsymbol{Q}$ corresponding to nonzero rows of $\boldsymbol{R}$ (except the first column $\boldsymbol{q}$ ) while $\boldsymbol{V}_{n}$ contains the columns of $\boldsymbol{Q}$ corresponding to zero rows of $\boldsymbol{R}$. Thus, $\operatorname{range}\left(\boldsymbol{T}_{n}^{\prime}\right)$ is the new adaptation space. Note that the removal of $t_{s}(\theta)$ not only completely eliminates signal cancellation at scenario $\theta$ but also generally reduces the signal cancellation capability at neighboring scenarios. Hence, usually only a few components of the original adaptation space need to be removed in order to satisfy the signal cancellation constraint over the interference scenario set $\mathcal{Q}$.

The multilevel design procedure described in [12] may also be employed to approximately minimize the number of components removed. Define a sequence of $L$ performance levels $\delta_{1}>\delta_{2}>\cdots>\delta_{L}=\delta_{o}$. We begin with $\delta_{1}$ and proceed to $\delta_{L}$. The performance levels are often chosen as a set of decreasing functions of the expected signal output power. As an example, we may choose $\delta_{1}=0.5 \boldsymbol{w}_{o}^{H} \boldsymbol{R}_{s} \boldsymbol{w}_{o}, \delta_{2}=$ $0.3 \boldsymbol{w}_{o}^{H} \boldsymbol{R}_{s} \boldsymbol{w}_{o}$, and $\delta_{3}=\delta_{o}=0.1 \boldsymbol{w}_{o}^{H} \boldsymbol{R}_{s} \boldsymbol{w}_{o}$. This constrains the worst case signal cancellation to be less that $10 \%$ of the signal output power. Values of $L$ ranging from two to four generally yield satisfactory results. At each level we only remove those components that cause signal cancellation levels higher than the current performance level. This multilevel design procedure essentially removes components from the original adaptation space based on their relative signal cancellation contributions. Components which result in the most severe signal cancellation are removed first: The following is a pseudo-code description of this procedure:

$$
\begin{aligned}
& \boldsymbol{T}_{n}=\boldsymbol{C}_{n} \\
& \text { for }=l=1 \text { to } L \\
& \text { for } k=1 \text { to } K \\
& \text { if }=P_{s c}\left(\boldsymbol{T}_{n}, \boldsymbol{\theta}_{k}\right)>\delta_{l} \\
& \text { perform } \mathrm{QR} \text { decomposition }\left[\begin{array}{ll}
\boldsymbol{T}_{s}(\boldsymbol{\theta}) & \boldsymbol{T}_{n}
\end{array}\right] \\
& =\left[\begin{array}{lll}
\boldsymbol{q} & \boldsymbol{T}_{n}^{\prime} & \boldsymbol{V}_{n}
\end{array}\right] \boldsymbol{R} \text { as in (44) } \\
& \text { end-of-if } \\
& \text { set } T_{n}=T_{n}^{\prime} \text {. } \\
& \text { end-loop-on- } k \\
& \text { end-loop-on- } l \text {. }
\end{aligned}
$$

Next, the nonadaptive weight vector $\boldsymbol{w}_{o}$ is modified to improve the beamformer's interference cancellation capability over the set of likely interference scenarios. That is, we choose a new nonadaptive weight vector $w_{q}$ that solves

$$
\begin{aligned}
& \min _{\boldsymbol{w}_{q}} \sum_{\boldsymbol{\theta} \in \mathcal{Q}} \boldsymbol{w}_{q}^{H}\left[\boldsymbol{R}_{n}(\boldsymbol{\theta})-\boldsymbol{R}_{n}(\boldsymbol{\theta}) \boldsymbol{T}_{n}\left(\boldsymbol{T}_{n}^{H} \boldsymbol{R}_{n}(\boldsymbol{\theta}) \boldsymbol{T}_{n}\right)^{-1}\right. \\
&\left.\cdot \boldsymbol{T}_{n}^{H} \boldsymbol{R}_{n}(\boldsymbol{\theta})\right] \boldsymbol{w}_{q}
\end{aligned}
$$

where $\boldsymbol{w}_{q}$ is constrained to be of the form $\boldsymbol{w}_{q}=\boldsymbol{w}_{o}-U_{n} \boldsymbol{w}_{m}$. Here $U_{n}$ is defined in (40) and $\boldsymbol{w}_{m}$ represents the $p-q$ available degrees of freedom in $w_{q}$. Since $w_{q}$ is nonadaptive, it is independent of $\boldsymbol{\theta}$ and (45) is equivalent to the quadratic minimization problem

$$
\min _{\boldsymbol{w}_{m}}\left(\boldsymbol{w}_{o}-\boldsymbol{U}_{n} \boldsymbol{w}_{m}\right)^{H} \boldsymbol{R}^{a v}\left(\boldsymbol{w}_{o}-\boldsymbol{U}_{n} \boldsymbol{w}_{m}\right)
$$

where

$$
\begin{aligned}
\boldsymbol{R}^{a v}= & \sum_{\boldsymbol{\theta} \in \mathcal{Q}}\left[\boldsymbol{R}_{n}(\boldsymbol{\theta})-\boldsymbol{R}_{n}(\boldsymbol{\theta}) \boldsymbol{T}_{n}\left(\boldsymbol{T}_{n}^{H} \boldsymbol{R}_{n}(\boldsymbol{\theta}) \boldsymbol{T}_{n}\right)^{-1}\right. \\
& \cdot \boldsymbol{T}_{n}^{H} \boldsymbol{R}_{n}(\boldsymbol{\theta})
\end{aligned}
$$

Solving (47) for $\boldsymbol{w}_{m}$ gives the nonadaptive weight vector

$$
\boldsymbol{w}_{q}=\boldsymbol{w}_{o}-\boldsymbol{U}_{n}\left(\boldsymbol{U}_{n}^{H} \boldsymbol{R}^{a v} \boldsymbol{U}_{n}\right)^{-1} \boldsymbol{U}_{n}^{H} \boldsymbol{R}^{a v} \boldsymbol{w}_{o}
$$

\section{PERFORMANCE ANALYSIS}

As noted in the previous section, reducing the number of adaptive degrees of freedom leads to loss in both signal and interference cancellation capabilities. In this section we assess the relative effects of partially adaptive beamforming on signal and interference cancellation from both time and frequency domain perspectives. This analysis does not consider the effects of modifying the beamformer's nonadaptive weight vector.

\section{A. Time Domain Analysis}

If $\boldsymbol{T}_{n}$ is chosen to satisfy (39), then

$$
\operatorname{range}\left(\boldsymbol{P}\left(\boldsymbol{C}_{n}\right)\right)=\operatorname{range}\left(\boldsymbol{P}\left(\boldsymbol{T}_{n}\right)\right) \oplus \operatorname{range}\left(\boldsymbol{z}_{s}\right) .
$$

Thus

$$
z_{n}=P\left(C_{n}\right) z_{n}=P\left(T_{n}\right) z_{n}+\frac{z_{s}^{H} z_{n}}{z_{s}^{H} z_{s}} z_{s}
$$

and

$$
\begin{aligned}
P_{n n}\left(\boldsymbol{C}_{n}\right) & =z_{n}^{H} z_{n}=z_{n}^{H} \boldsymbol{P}\left(\boldsymbol{T}_{n}\right) z_{n}+\frac{\left|z_{s}^{H} z_{n}\right|^{2}}{z_{s}^{H} z_{s}} \\
& =P_{n n}\left(\boldsymbol{T}_{n}\right)+P_{n n}\left(\boldsymbol{C}_{n}\right) \cos ^{2} \phi_{z}
\end{aligned}
$$

where $\phi_{z}$ is the angle between $z_{n}$ and $z_{s}$. We then obtain

$$
\begin{aligned}
P_{n n}\left(\boldsymbol{T}_{n}\right) & =P_{n n}\left(\boldsymbol{C}_{n}\right)\left(1-\cos ^{2} \phi_{z}\right) \\
P_{s c}\left(\boldsymbol{T}_{n}\right) & =0 .
\end{aligned}
$$

The partially adaptive beamformer experiences very little loss in interference cancellation if

$$
\cos ^{2} \phi_{z} \approx 0 \text {. }
$$

The success of partially adaptive beamforming depends on the relative orientation of the vectors $z_{n}$ and $z_{s}$. If $z_{n}$ and $z_{s}$ are orthogonal, then choosing $\boldsymbol{T}_{n}$ to satisfy (39) yields $\boldsymbol{z}_{s} \in \operatorname{range}^{\perp}\left(\boldsymbol{P}\left(\boldsymbol{T}_{n}\right)\right)$ and $\boldsymbol{z}_{n} \in \operatorname{range}\left(\boldsymbol{P}\left(\boldsymbol{T}_{n}\right)\right)$. Thus, $\boldsymbol{P}\left(\boldsymbol{T}_{n}\right) z_{s}=\boldsymbol{o}$ and $\boldsymbol{P}\left(\boldsymbol{T}_{n}\right) z_{n}=z_{n}$. In this case, fully adaptive interference cancellation $\left(P_{n n}\left(\boldsymbol{T}_{n}\right)=P_{n n}\left(\boldsymbol{C}_{n}\right)\right)$ is obtained with zero signal cancellation $\left(P_{s c}\left(\boldsymbol{T}_{n}\right)=0\right)$. On the other hand, if $z_{n}$ and $z_{s}$ are collinear, then any choice for $T_{n}$ 
will reduce $P_{n n}$ and $P_{s c}$ equally and the partially adaptive approach does not yield any performance improvement in the sense that $P_{n n}\left(\boldsymbol{T}_{n}\right) / P_{s c}\left(\boldsymbol{T}_{n}\right)=P_{n n}\left(\boldsymbol{C}_{n}\right) / P_{s c}\left(\boldsymbol{C}_{n}\right)$. In practice, $z_{n}$ and $z_{s}$ will generally be neither orthogonal nor collinear; the partially adaptive beamformer's signal cancellation capability is completely disabled while its interference cancellation capability is reduced by the factor $1-\cos ^{2} \phi_{z}$. Note that

$$
\cos ^{2} \phi_{z}=\frac{\left|z_{n}^{H} z_{s}\right|^{2}}{z_{n}^{H} z_{n} z_{s}^{H} z_{s}}=\frac{\left|P_{n s}\left(C_{n}\right)\right|^{2}}{P_{n n}\left(C_{n}\right) P_{s c}\left(C_{n}\right)}
$$

where $P_{n s}$ is defined in (31).

Recall that $n_{n}$ and $n_{s}$ are the components of the adaptive branch in the GSC used to cancel the interferece $n_{o}$ and signal $s$, respectively, at the nonadaptive beamformer output. Let $r\left(n_{n}, n_{s}\right)$ be the cross correlation coefficient between $n_{n}$ and $n_{s}$ for a fully adaptive beamformer $\left(T_{n}=C_{n}\right)$, i.e.

$$
r\left(n_{n}, n_{s}\right)=\frac{E\left\{n_{n}^{*} n_{s}\right\}}{\sqrt{E\left\{n_{n}^{*} n_{n}\right\} E\left\{n_{s}^{*} n_{s}\right\}}} .
$$

Straightforward calculation shows

$$
\cos ^{2} \phi_{z}=\left|r\left(n_{n}, n_{s}\right)\right|^{2}
$$

Hence, good interference cancellation can be obtained with zero signal cancellation if the components $n_{s}$ and $n_{n}$ from the adaptive branch of the fully adaptive beamformer are weakly correlated. This result is intuitively satisfying. If $n_{n}$ and $n_{s}$ are uncorrelated, then $\boldsymbol{T}_{n}$ can be chosen so that only $n_{n}$ passes through the adaptive branch. This results in fully adaptive interference cancellation and zero signal cancellation. In contrast, if $n_{n}$ and $n_{s}$ are correlated, then the portion of $n_{n}$ that is correlated with $n_{s}$ will not be present at the adaptive branch output when $\boldsymbol{T}_{n}$ is chosen to prevent signal cancellation and less of the interference at the nonadaptive beamformer output is cancelled.

If there is strong correlation between an interferer and the desired signal such that complete signal cancellation occurs $\left(s \approx n_{s}\right)$ and the beamformer attains nearly complete interference cancellation $\left(n_{o} \approx n_{n}\right)$, then we obtain

$$
\cos ^{2} \phi_{z} \approx \frac{\left|\boldsymbol{w}_{o}^{H} \boldsymbol{R}_{n s} \boldsymbol{w}_{o}\right|^{2}}{P_{n o} P_{s}}
$$

by substituting $n_{o}$ and $s$ for $n_{n}$ and $n_{s}$ in (56) and (57). Equation (58) indicates that the relative magnitude of $\boldsymbol{w}_{o}^{H} \boldsymbol{R}_{n, s} \boldsymbol{w}_{o}=$ $E\left\{n_{o} s^{*}\right\}$ is the determining factor for the potential effectiveness of the partially adaptive beamforming approach. If the correlation between $n_{o}$ and $s$ is small relative to the total power in $n_{o}$ and $s$, then an effective partially adaptive beamformer can be designed.

\section{B. Frequency Domain Analysis}

An alternate perspective of the effectiveness of the partially adaptive beamforming approach is obtained by analyzing the capability of the adaptive branch frequency response to match the nonadaptive branch frequency response.

Assume all signals of interest lie in the frequency band in $\left[\omega_{1}, \omega_{2}\right]$ and for ease of exposition that the environment consists of the desired signal and a single correlated interferer arriving from directions $\theta_{s}$ and $\theta_{c}$, respectively, in white noise of power $\sigma^{2}$. (This analysis is extended in the Appendix to include additional uncorrelated interferers.) Let $S^{2}(\omega)$ and $C^{2}(\omega)$ be the power spectral densities of the desired signal and correlated interferer with $S(\omega)$ and $C(\omega)$ their positive square roots and $\rho(\omega)$ be the cross correlation coefficient between signal and interferer $(|\rho(\omega)| \leq 1)$. Under these assumptions

$$
\begin{aligned}
\boldsymbol{R}_{n} & =\int_{\omega_{1}}^{\omega_{2}} C^{2}(\omega) \boldsymbol{d}\left(\omega, \theta_{c}\right) \boldsymbol{d}^{H}\left(\omega, \theta_{c}\right) d \omega+\sigma^{2} \boldsymbol{I} \\
\boldsymbol{R}_{n s} & =\int_{\omega_{1}}^{\omega_{2}} \rho(\omega) C(\omega) S(\omega) \boldsymbol{d}\left(\omega, \theta_{c}\right) \boldsymbol{d}^{H}\left(\omega, \theta_{s}\right) d \omega
\end{aligned}
$$

where $d(\omega, \theta)$ denotes the array response vector [6] in direction $\theta$ and at frequency $\omega . P_{n n}$ and $P_{s c}$ are now rewritten as

$$
\begin{aligned}
P_{n n}= & \int_{\omega_{1}}^{\omega_{2}} q_{n}(\omega) \boldsymbol{v}^{H}(\omega) d \omega\left[\int_{\omega_{1}}^{\omega_{2}} \boldsymbol{v}(\omega) \boldsymbol{v}^{H}(\omega) d \omega+\sigma^{2} \boldsymbol{I}\right]^{-1} \\
& \cdot \int_{\omega_{1}}^{\omega_{2}} \boldsymbol{v}(\omega) q_{n}^{*}(\omega) d \omega \\
P_{s c}= & \int_{\omega_{1}}^{\omega_{2}} q_{s}(\omega) \boldsymbol{v}^{H}(\omega) d \omega\left[\int_{\omega_{1}}^{\omega_{2}} \boldsymbol{v}(\omega) \boldsymbol{v}^{H}(\omega) d \omega+\sigma^{2} \boldsymbol{I}\right]^{-1} \\
& \cdot \int_{\omega_{1}}^{\omega_{2}} \boldsymbol{v}(\omega) q_{s}^{*}(\omega) d \omega
\end{aligned}
$$

where

$$
\begin{aligned}
q_{n}(\omega) & =C(\omega) \boldsymbol{w}_{o}^{H} d\left(\omega, \theta_{c}\right), \\
q_{s}(\omega) & =\rho^{*}(\omega) S(\omega) \boldsymbol{w}_{o}^{H} d\left(\omega, \theta_{s}\right), \\
\boldsymbol{v}(\omega) & =C(\omega) \boldsymbol{T}_{n}^{H} d\left(\omega, \theta_{c}\right) .
\end{aligned}
$$

$v(\omega)$ and $q_{n}(\omega)$ represent the frequency content of the correlated interferer at the output of the blocking matrix $T_{n}$ and nonadaptive beamformer $\boldsymbol{w}_{o}$, respectively. $q_{s}(\omega)$ is the frequency content of the portion of the desired signal at the nonadaptive beamformer output that is correlated with the interferer.

The expressions for $P_{n n}$ and $P_{s c}$ are simplified by representing $v(\omega)$ in terms of an orthonormal set of basis functions. Let $\boldsymbol{R}_{c}=\int_{\omega_{1}}^{\omega_{2}} \boldsymbol{v}(\omega) \boldsymbol{v}^{H}(\omega) d \omega$ have the eigendecomposition

$$
\boldsymbol{R}_{c}=\boldsymbol{V} \boldsymbol{\Sigma}^{2} \boldsymbol{V}^{H}
$$

where $\Sigma^{2}$ is a diagonal matrix of eigenvalues and the columns of $\boldsymbol{V}$ are the corresponding eigenvectors. We assume the eigenvalues are ordered

$$
\sigma_{1}^{2} \geq \sigma_{2}^{2} \geq \cdots \geq \sigma_{p}^{2} \geq 0 .
$$

Define the transformed basis for the frequency content of the blocking matrix output

$$
\boldsymbol{u}(\omega)=\boldsymbol{\Sigma}^{-1} \boldsymbol{V}^{H} \boldsymbol{v}(\omega)
$$

The case where some of the eigenvalues are zero is easily accommodated by replacing the inverse in (68) with a psuedoinverse. Note that a zero eigenvalue indicates that $v(\omega)$ 
has no component in the space spanned by the corresponding eigenvector. The orthonormality of $\boldsymbol{u}(\omega)$ is easily verified

$$
\begin{aligned}
\int_{\omega_{1}}^{\omega_{2}} \boldsymbol{u}(\omega) \boldsymbol{u}^{H}(\omega) d \omega & =\boldsymbol{\Sigma}^{-1} \boldsymbol{V}^{H} \int_{\omega_{1}}^{\omega_{2}} \boldsymbol{v}(\omega) \boldsymbol{v}^{H}(\omega) d \omega \boldsymbol{V} \boldsymbol{\Sigma} \\
& =\boldsymbol{\Sigma}^{-1} \boldsymbol{V}^{H} \boldsymbol{R}_{c} \boldsymbol{V} \boldsymbol{\Sigma}=\boldsymbol{I}
\end{aligned}
$$

Substituting $V \boldsymbol{\Sigma u}(\omega)$ for $v(\omega)$ in (61) and (62) yields

$$
\begin{aligned}
P_{n n} & =\boldsymbol{h}_{n}^{H} \boldsymbol{\Sigma} \boldsymbol{V}^{H}\left[\boldsymbol{V}\left(\boldsymbol{\Sigma}^{2}+\sigma^{2} \boldsymbol{I}\right) \boldsymbol{V}^{H}\right]^{-1} \boldsymbol{V} \boldsymbol{\Sigma} \boldsymbol{h}_{n} \\
P_{s c} & =\boldsymbol{h}_{s}^{H} \boldsymbol{\Sigma} \boldsymbol{V}^{H}\left[\boldsymbol{V}\left(\boldsymbol{\Sigma}^{2}+\sigma^{2} \boldsymbol{I}\right) \boldsymbol{V}^{H}\right]^{-1} \boldsymbol{V} \boldsymbol{\Sigma} \boldsymbol{h}_{s}
\end{aligned}
$$

where

$$
\begin{aligned}
& \boldsymbol{h}_{n}=\int_{\omega_{1}}^{\omega_{2}} \boldsymbol{u}(\omega) q_{n}^{*}(\omega) d \omega \\
& \boldsymbol{h}_{s}=\int_{\omega_{1}}^{\omega_{2}} \boldsymbol{u}(\omega) q_{s}^{*}(\omega) d \omega .
\end{aligned}
$$

Simplifying (70) and (71), we obtain

$$
\begin{aligned}
P_{n n} & =\sum_{i=1}^{p} \frac{\sigma_{i}^{2}}{\sigma_{i}^{2}+\sigma^{2}}\left|h_{n i}\right|^{2} \\
P_{s c} & =\sum_{i=1}^{p} \frac{\sigma_{i}^{2}}{\sigma_{i}^{2}+\sigma^{2}}\left|h_{s i}\right|^{2} .
\end{aligned}
$$

If we assume that $\boldsymbol{R}_{c}$ is approximately rank $r$, that is, the eigenvalues satisfy

$$
\sigma_{1}^{2} \geq \sigma_{2}^{2} \geq \cdots \geq \sigma_{r}^{2} \gg \sigma^{2} \gg \sigma_{r+1}^{2} \geq \cdots \geq \sigma_{p}^{2}
$$

then (74) and (75) are

$$
\begin{gathered}
P_{n n} \approx\left(\boldsymbol{h}_{n}^{r}\right)^{H} \boldsymbol{h}_{n}^{r} \\
P_{s c} \approx\left(\boldsymbol{h}_{s}^{r}\right)^{H} \boldsymbol{h}_{s}^{r}
\end{gathered}
$$

where $\boldsymbol{h}_{n}^{r}$ and $\boldsymbol{h}_{\boldsymbol{s}}^{r}$ contain the first $r$ elements of $\boldsymbol{h}_{n}$ and $\boldsymbol{h}_{\boldsymbol{s}}$, respectively.

The vectors $h_{n}$ and $h_{s}$ are the coordinates of $q_{n}(\omega)$ and $q_{s}(\omega)$ in the space spanned by the components $\left\{u_{i}(\omega)\right\}$ of the vector function $\boldsymbol{u}(\omega)$. Hence, the interference cancellation level is determined by the projection of the nonadaptive branch interference frequency content onto the space spanned by the adaptive branch interference frequency content. Similarly, the signal cancellation level is determined by the projection of the portion of the signal frequency content in the nonadaptive branch that is correlated with the interferer onto the space spanned by the adaptive branch interference frequency content. The goal is to choose $\boldsymbol{T}_{n}$ and $\boldsymbol{w}_{o}$ so that there is a good match between the nonadaptive and adaptive branch interference frequency content and a poor match between the adaptive branch interference frequency content and the correlated portion of the signal in the nonadaptive branch. Replacing $\boldsymbol{C}_{n}$ with $\boldsymbol{T}_{n}$ shrinks the space spanned by the basis $\boldsymbol{u}(\omega)$. Modification of $\boldsymbol{w}_{o}$ changes the projections of $q_{n}(\omega)$ and $q_{s}(\omega)$ onto this space.

Similar to (77) and (78)

$$
P_{n s} \approx\left(\boldsymbol{h}_{n}^{r}\right)^{H} \boldsymbol{h}_{s}^{r}
$$

Hence, assuming $\boldsymbol{T}_{n}=\boldsymbol{C}_{n}$ and $\boldsymbol{R}_{c}$ is rank $r$, (55) is rewritten as

$$
\cos ^{2} \phi_{z} \approx \frac{\left|\left(\boldsymbol{h}_{n}^{r}\right)^{H} \boldsymbol{h}_{s}^{r}\right|^{2}}{\left(\boldsymbol{h}_{n}^{r}\right)^{H} \boldsymbol{h}_{n}^{r}\left(\boldsymbol{h}_{s}^{r}\right)^{H} \boldsymbol{h}_{s}^{r}} .
$$

Here we see that $\cos ^{2} \phi_{z}$ corresponds to the cosine squared of the angle between $\boldsymbol{h}_{n}^{r}$ and $\boldsymbol{h}_{s}^{r}$.

If $q_{n}(\omega)=\alpha q_{s}(\omega)$ for some constant $\alpha$, then $\boldsymbol{h}_{n}=\alpha^{*} \boldsymbol{h}_{\boldsymbol{s}}$. In this case $\cos ^{2} \phi_{z} \approx 1$ and partially adaptive beamforming is ineffective. In particular, if the signal and interferer are narrowband, i.e., $\omega_{1}=\omega_{2}$, we have $q_{n}\left(\omega_{1}\right)=\alpha q_{s}\left(\omega_{1}\right)$. Indeed, since $\boldsymbol{R}_{c}=\boldsymbol{v}\left(\omega_{1}\right) \boldsymbol{v}^{H}\left(\omega_{1}\right)$ is rank one in this case, it is easy to verify that $u(\omega)=1$. Consequently, $P_{n n}$ and $P_{s c}$ are independent of the adaptation space represented by $\boldsymbol{T}_{n}$ and we conclude that partially adaptive beamforming cannot succeed with narrowband signals.

In the case of strong correlated interference $|\rho(\omega)|^{2} \approx 1$. Again assuming $T_{n}=C_{n}$ and nearly complete signal and interference cancellation, we have

$$
\begin{aligned}
P_{n o}= & \int_{\omega_{1}}^{\omega_{2}} C^{2}(\omega)\left|\boldsymbol{w}_{o}^{H} d\left(\omega, \theta_{c}\right)\right|^{2} d \omega \\
= & \int_{\omega_{1}}^{\omega_{2}}\left|q_{n}(\omega)\right|^{2} d \omega \\
P_{s}= & \int_{\omega_{1}}^{\omega_{2}} S^{2}(\omega)\left|\boldsymbol{w}_{o}^{H} d\left(\omega, \theta_{s}\right)\right|^{2} d \omega \\
& \approx \int_{\omega_{1}}^{\omega_{2}}|\rho(\omega)|^{2} S^{2}(\omega)\left|\boldsymbol{w}_{o}^{H} d\left(\omega, \theta_{s}\right)\right|^{2} d \omega \\
= & \int_{\omega_{1}}^{\omega_{2}}\left|q_{s}(\omega)\right|^{2} d \omega \\
\boldsymbol{w}_{o}^{H} \boldsymbol{R}_{n s} \boldsymbol{w}_{o}= & \int_{\omega_{1}}^{\omega_{2}} \rho(\omega) C(\omega) S(\omega) \boldsymbol{w}_{o}^{H} d\left(\omega, \theta_{c}\right) d^{H}\left(\omega, \theta_{s}\right) \\
& \cdot \boldsymbol{w}_{o} d \omega=\int_{\omega_{1}}^{\omega_{2}} q_{n}(\omega) q_{s}^{*}(\omega) d \omega
\end{aligned}
$$

and (58) is expressed in the alternate form

$$
\cos ^{2} \phi_{z} \approx \frac{\left|\int_{\omega_{1}}^{\omega_{2}} q_{n}(\omega) q_{s}^{*}(\omega) d \omega\right|^{2}}{\int_{\omega_{1}}^{\omega_{2}}\left|q_{n}(\omega)\right|^{2} d \omega \int_{\omega_{1}}^{\omega_{2}}\left|q_{s}(\omega)\right|^{2} d \omega}
$$

Here $\cos ^{2} \phi_{z}$ corresponds to the cosine squared of the angle between the functions $q_{n}(\omega)$ and $q_{s}(\omega)$. Equation (84) also follows from (80). If nearly complete signal and interference cancellation is attained, then both $q_{n}(\omega)$ and $q_{s}(\omega)$ lie almost entirely in the space spanned by the elements of $u(\omega) . u(\omega)$ is an orthonormal basis so the inner product involving $\boldsymbol{h}_{n}^{r}$ and $\boldsymbol{h}_{\boldsymbol{s}}^{r}$ are equivalent to the inner product involving $q_{n}(\omega)$ and $q_{s}(\omega)$.

\section{EXAMPLES}

The performance of two partially adaptive beamformers obtained through the multilevel point design (PD) procedures described in Section IV is evaluated in this section and compared to that of a fully adaptive beamformer and the CSS beamformer. 
The array employed has nine sensors in an equally spaced linear geometry with nine tap FIR filters in each sensor channel, resulting in a total of 81 weights. The uniform structure is chosen for convenience, and is not required by the partially adaptive beamforming technique. The tap spacing is chosen in accordance with the Nyquist sampling rate so that frequency is normalized on the interval $[0, \pi]$, i.e., the sampling time is normalized to 1 . The distance between adjacent sensors is one-half the wavelength of the highest signal frequency. All signals are assumed to lie in the frequency band $[9 / 11 \pi, \pi]$. The desired signal arrives from the direction perpendicular to the array, i.e., at a direction sine of 0 . Five of the available 81 degrees of freedom are used to provide a unit gain and linear phase response in the desired signal direction. The interference spectra are assumed white on $[9 / 11 \pi, \pi]$. The desired output signal power (11) is normalized to 1 in this example.

\section{A. Fully Adaptive Beamforming}

Here we illustrate the signal cancellation phenomenon when fully adaptive beamforming (FAB) is employed in a correlated interference scenario. Assume there are two uncorrelated interferers with direction sines $0.45\left(26.74^{\circ}\right)$ and $-0.55\left(-33.37^{\circ}\right)$, each of power level 10 relative to signal power spectral density. A correlated interferer which is a delayed $(0.1 \mathrm{sec})$ and scaled (0.8) version of the desired signal is also present at direction sine $-0.22\left(-12.71^{\circ}\right)$. The background white noise level is 0.01 .

If fully adaptive beamforming is employed, the beamformer output power is $P_{o}=0.0012$ which indicates severe signal cancellation. In this case, $P_{n o}=0.2357, P_{n n}=0.2353$, and $P_{s c}=0.9989$. Therefore, fully adaptive beamforming not only cancels the uncorrelated interferers but cancels the desired signal as well. The corresponding $M S E=0.9993$. This signal cancellation is also evident in the adapted beampattern. As shown in Fig. 3, unit gain is obtained at direction sine 0 as the result of linear constraints. Two nulls are formed at direction sines -0.55 and 0.45 , corresponding to the two uncorrelated interferers. The beamformer does not put a null at direction sine -0.22 , the correlated interferer's direction. Instead, the correlated interferer is amplified by $1 / 0.8$ and phase shifted appropriately to cancel the desired signal.

\section{B. Partially Adaptive Beamforming}

A partially adaptive beamformer is now designed as described in Section IV. For the design process, two uncorrelated interferers are assumed to be present with direction sines in the intervals $[0.21]\left(\left[11.54^{\circ}, 90^{\circ}\right]\right)$ and $[-1,-0.5]\left[-90^{\circ},-30^{\circ}\right]$, respectively. The correlated interferer's direction sine is assumed to be in the interval $[-0.4,-0.2]\left[-23.58^{\circ},-11.54^{\circ}\right]$. All other interference information is assumed known. Using a 2-level ( $\delta_{1}=0.3, \delta_{2}=0.1$ ) point design procedure, a partially adaptive beamformer of dimension 71 is obtained. Evaluating the partially adaptive beamformer for the same interference scenario used for the fully adaptive beamformer, we have $P_{n o}=0.2357, P_{n n}=0.2315$, and $P_{s c}=0.0001$, resulting in $M S E=0.0103$. The beamformer output power is $P_{o}=$ 0.9047. Therefore, severe signal cancellation is avoided at the

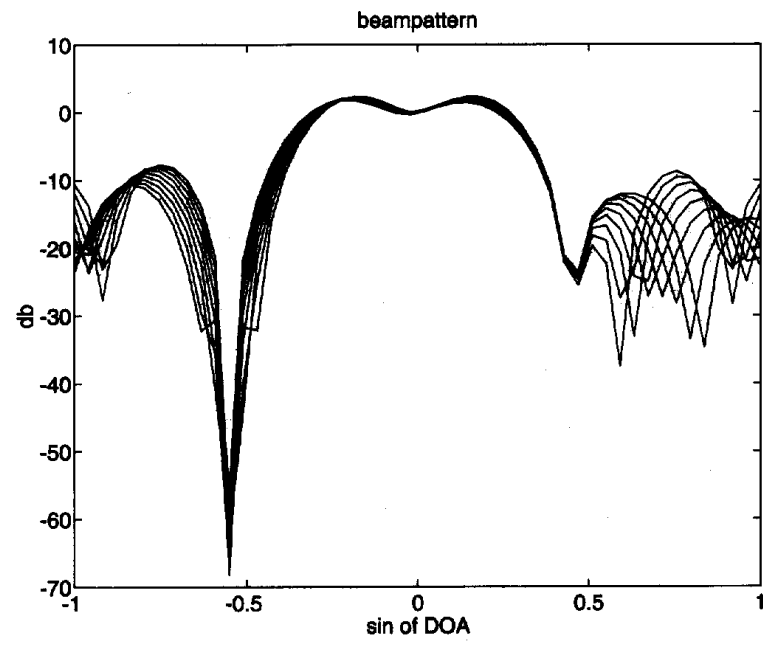

Fig. 3. Beampattern of fully adaptive beamformer. Each line represents the response at a different frequency in the band $9 / 11 \pi, \pi]$.

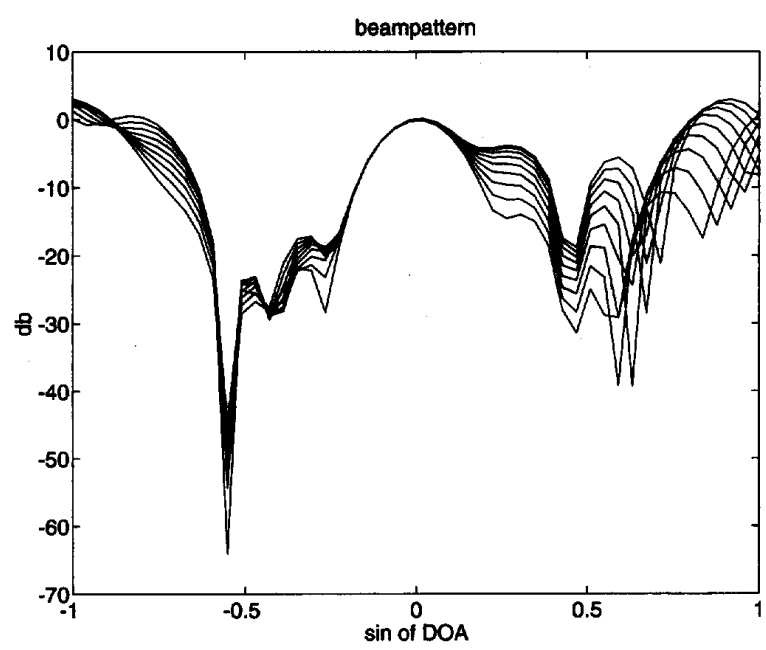

Fig. 4. Beampattern of partially adaptive beamformer. Each line represents the response at a different frequency in the band $[9 / 11 \pi, \pi]$.

expense of a slight degradation in interference cancellation capability. The adapted beampattern shown in Fig. 4 indicates that an approximate null is placed at the direction sine of each interferer.

The partially adaptive beamformer performance is further evaluated using 1500 interference scenarios uniformly distributed on the intervals assumed for design. The maximum $M S E$ is 0.0382 and the average is 0.0070 . Hence, the partially adaptive beamformer performs very well for all assumed interference scenarios.

\section{Comparison with Frequency Domain Averaging Method}

The partially adaptive beamforming (PAB) technique proposed here is effective for broadband signals. An alternate technique for correlation reduction in broadband signal environments is the frequency domain averaging or CSS technique 
TABLE I

MSE Performance as a Function of CoRrelated INTERfERer TIME DELAY for PARTIALly ADAPTIVE (PAB) CSS, AND FULLY ADAPTIVE (FAB) BEAMFORMERS

\begin{tabular}{||c|c|c|c|c|c|c||}
\hline & \multicolumn{2}{|c|}{ PAB $M S E$} & \multicolumn{2}{c|}{ CSS $M S E$} & \multicolumn{2}{c|}{ FAB $M S E$} \\
\hline delay & $\max$ & ave & $\max$ & ave & $\max$ & ave \\
\hline 0.0000 & 0.0154 & 0.0080 & 1.0590 & 1.0167 & 1.0000 & 1.0000 \\
\hline 0.1111 & 0.0160 & 0.0082 & 1.01445 & 1.0027 & 1.0000 & 1.0060 \\
\hline 0.2222 & 0.0169 & 0.0085 & 0.9932 & 0.9605 & 1.0000 & 0.9599 \\
\hline 0.3333 & 0.0174 & 0.0086 & 0.9477 & 0.8897 & 0.9995 & 0.9994 \\
\hline 0.4444 & 0.0174 & 0.0088 & 0.8935 & 0.7916 & 0.9980 & 0.9974 \\
\hline 0.5556 & 0.0171 & 0.0091 & 0.8226 & 0.6719 & 0.9937 & 0.9922 \\
\hline 0.6667 & 0.0164 & 0.0093 & 0.7328 & 0.5388 & 0.9843 & 0.9810 \\
\hline 0.7778 & 0.0157 & 0.0093 & 0.6284 & 0.4096 & 0.9667 & 0.9607 \\
\hline 0.8889 & 0.0159 & 0.0094 & 0.5146 & 0.2891 & 0.9374 & 0.9278 \\
\hline 10.0000 & 0.0171 & 0.0100 & 0.4046 & 0.1996 & 0.8933 & 0.8797 \\
\hline \hline
\end{tabular}

[4]. Here the performance of adaptive beamformers designed using these two techniques are compared.

As is shown in the previous example, an effective partially adaptive beamformer can be designed for a wide range of interference scenarios. However, the CSS method requires preliminary estimates of the interferer and signal directions. These estimates result in much smaller direction-of-arrival (DOA) intervals than those used for the previous example. Therefore, a new set of interference scenarios based on assumed DOA estimates are used here to design the partially adaptive beamformer. Three unity power interferers are embedded in $-30 \mathrm{~dB}$ white noise: two uncorrelated interferers (one with incident angle in the interval $\left[-52^{\circ}\right.$, $\left.-48^{\circ}\right]$ and the other in the interval $\left.\left[8^{\circ}, 12^{\circ}\right]\right)$ and a correlated interferer whose incident angle is in the interval $\left[28^{\circ}, 32^{\circ}\right]$. The latter is a delayed version of the desired signal. The possible time delay of the correlated interferer is assumed to range from 0 to 10 time units.

The CSS method uses $-52^{\circ},-48^{\circ},-1^{\circ}, 1^{\circ}, 8^{\circ}, 12^{\circ}, 28^{\circ}$, and $32^{\circ}$ as the DOA estimates. In order to construct a square $(9 \times 9)$ full rank focusing transformation matrix, a fictitious signal from direction $80^{\circ}$ is also included. While a time domain approximation of the CSS transformation is proposed in [13], we use the exact frequency domain transformation to optimize the CSS method performance. Note that the exact transformation cannot be physically implemented.

A 62-dimensional partially adaptive beamformer is designed for a set of likely interference scenarios that consists of all combinations of three interferers from the same intervals used by the CSS processor $\left(\left[-52^{\circ},-48^{\circ}\right],\left[8^{\circ}, 12^{\circ}\right],\left[28^{\circ}, 32^{\circ}\right]\right)$ with the correlated interferer's time delay in the range $[0,10]$.

Partially adaptive, CSS, and fully adaptive beamformer performance is evaluated at 1250 distinct interference scenarios obtained by varying uniformly within the set of likely interference scenarios. The result is summarized in Table I as a function of the time delay. The average $M S E$ 's for each time delay value are obtained by averaging over 125 scenarios.

The partially adaptive beamforming technique significantly outperforms the frequency domain averaging technique over this range of time delays between the desired signal and correlated interferer. The frequency domain averaging technique avoids severe signal cancellation only when the time delays are relatively long. This is because that the frequency domain averaging technique requires the time delay to be much longer than the reciprocal of the signal bandwidth $(=11 / 2 \pi=$ 1.7507 in this example) even when the exact interference directions are available [4]. The partially adaptive beamformer is effective for situations where the time delay between the desired signal and interferers is very short, even where the time delay is zero. Furthermore, it accommodates a wider variation in the interferer's DOA's.

\section{SUMMARY}

In this paper, we propose a partially adaptive beamforming technique to prevent signal cancellation in the presence of correlated interferers. By decomposing the beamformer's output mean squared error into an interference mean squared error term and a signal cancellation term, a partially adaptive beamformer design criterion is proposed. This criterion constrains the beamformer signal cancellation level for a set of likely interference scenarios. A procedure is given for designing a partially adaptive beamformer that satisfies the criterion. Analyses in both time domain and frequency domain reveal that the effectiveness of the approach is dependent on the cosine squared of the angle between interference and signal vectors. This method is only effective for broadband signal environments. Simulations demonstrate that the partially adaptive beamforming approach is effective even when the time delay between the desired signal and correlated interferer is very short.

\section{APPENDIX}

Here we extend the frequency domain analysis to include additional uncorrelated interferers. For simplicity, we only assume one additional uncorrelated interferer from direction $\theta_{n}$ with power spectral density $N^{2}(\omega)$. The case of multiple noncorrelated interferers follows directly. The interference covariance matrix now becomes

$$
\begin{aligned}
\boldsymbol{R}_{n}= & \int_{\omega_{1}}^{\omega_{2}} C^{2}(\omega) \boldsymbol{d}\left(\omega, \theta_{c}\right) \boldsymbol{d}^{H}\left(\omega, \theta_{c}\right)+N^{2}(\omega) \boldsymbol{d}\left(\omega, \theta_{n}\right) \\
& \cdot \boldsymbol{d}^{H}\left(\omega, \theta_{n}\right) d \omega+\sigma^{2} \boldsymbol{I} .
\end{aligned}
$$

Let the eigendecomposition of

$$
\begin{gathered}
\int_{\omega_{1}}^{\omega_{2}} C^{2}(\omega) \boldsymbol{T}_{n}^{H} \boldsymbol{d}\left(\omega, \theta_{c}\right) \boldsymbol{d}^{H}\left(\omega, \theta_{c}\right) \boldsymbol{T}_{n}+N^{2}(\omega) \boldsymbol{T}_{n}^{H} \\
\cdot d\left(\omega, \theta_{n}\right) \boldsymbol{d}^{H}\left(\omega, \theta_{n}\right) \boldsymbol{T}_{n} d \omega
\end{gathered}
$$

be $\boldsymbol{V} \boldsymbol{\Sigma}^{2} \boldsymbol{V}^{H}$ and define

$$
\begin{aligned}
\boldsymbol{q}_{n}(\omega)= & {\left[C(\omega) \boldsymbol{w}_{o}^{H} d\left(\omega, \theta_{c}\right) ; N(\omega) \boldsymbol{w}_{o}^{H} \boldsymbol{d}\left(\omega, \theta_{n}\right)\right] } \\
\boldsymbol{q}_{s}(\omega)= & {\left[\rho^{*}(\omega) \boldsymbol{w}_{o}^{H} \boldsymbol{d}\left(\omega, \theta_{s}\right) ; 0\right] } \\
\boldsymbol{U}(\omega)= & {\left[\boldsymbol{u}_{c}(\omega) ; \boldsymbol{u}_{n}(\omega)\right]=\boldsymbol{\Sigma}^{-1} \boldsymbol{V}^{H}\left[C(\omega) \boldsymbol{T}_{n}^{H} \boldsymbol{d}\left(\omega, \theta_{c}\right)\right.} \\
& \left.N(\omega) \boldsymbol{T}_{n}^{H} \boldsymbol{d}\left(\omega, \theta_{n}\right)\right]
\end{aligned}
$$

It is easy to verify that

$$
\int_{\omega_{1}}^{\omega_{2}} U(\omega) U^{H}(\omega)=I_{r}
$$


Therefore, as before, we have

$$
\begin{gathered}
P_{n n}=\boldsymbol{h}_{n}^{H} \operatorname{diag}\left(\frac{\sigma_{i}^{2}}{\sigma_{i}^{2}+\sigma^{2}}\right) \boldsymbol{h}_{n} \\
P_{s c}=\boldsymbol{h}_{s}^{H} \operatorname{diag}\left(\frac{\sigma_{i}^{2}}{\sigma_{i}^{2}+\sigma^{2}}\right) \boldsymbol{h}_{s}
\end{gathered}
$$

where

$$
\begin{aligned}
& \boldsymbol{h}_{n}=\int_{\omega_{1}}^{\omega_{2}} \boldsymbol{U}(\omega) \boldsymbol{q}_{n}^{H}(\omega) d \omega \\
& \boldsymbol{h}_{s}=\int_{\omega_{1}}^{\omega_{2}} \boldsymbol{U}(\omega) \boldsymbol{q}_{s}^{H}(\omega) d \omega
\end{aligned}
$$

and $\operatorname{diag}\left(\sigma_{i}^{2} /\left[\sigma_{i}^{2}+\sigma^{2}\right]\right)$ denotes a diagonal matrix whose $i$ th diagonal element is $\sigma_{i}^{2} /\left(\sigma_{i}^{2}+\sigma^{2}\right)$.

\section{REFERENCES}

[1] K. Duvall, "Signal cancellation in adaptive arrays: Phenomena and a remedy," Ph.D. dissertation, Dept. Elec. Eng., Stanford Univ., Sept. 1983.

[2] T. J. Shan and T. Kailath, “Adaptive beamforming for correlated signals and interference," IEEE Trans. Acoust. Speech, Signal Processing, vol. ASSP-33, pp. 527-536, June 1985.

[3] Y. L. Su, T. J. Shan, and B. Widrow, "Parallel spatial processing: A cure for signal cancellation in adaptive arrays," IEEE Trans. Antennas Propagat., vol. AP-34, pp. 347-355, Mar. 1986.

[4] J. F. Yang and M. Kaveh, "Coherent signal-subspace transformation beamformer," IEE Proc., vol. 137, no. 4, pp. 267-275, Aug. 1990.

[5] M. Lu and Z. He, "Adaptive beamforming using split-polarity transformation for correlated signal and interference," IEEE Trans. Antennas Propagat., vol. 41, pp. 314-324, Mar. 1993.

[6] B. D. Van Veen, "Minimum variance beamforming," S. Haykin and A. Steinhardt, Eds., in Adaptive Radar Detection and Estimation. New York: Wiley, 1992, ch. 4.

[7] L. J. Griffiths and C. W. Jim, "An alternative approach to linearly constrained adaptive beamforming," IEEE Trans. Antennas Propagat., vol. AP-30, pp. 27-34, Jan. 1982.

[8] D. R. Morgan, "Partially adaptive array techniques," IEEE Trans. Antennas Propagat, vol. AP-26, pp. 823-833, Nov. 1978.

[9]. W. F. Gabriel, "Using spectral estimation techniques in adaptive processing antenna systems," IEEE Trans. Antennas Propagat., vol. AP-34, pp. 291-300, Mar. 1986 .
[10] B. D. Van Veen, "Optimization of quiescent response in partially adaptive beamformers," IEEE Trans. Acoust., Speech, Signal Processing, vol. 38, pp. 471-477, Mar. 1990.

[11] F. Qian and B. D. Van Veen, "Coherent interference suppression via partially adaptive beamforming," in Proc. 1992 ICASSP, 1992, vol. IV, pp. $441-444$.

[12] F. Qian and B. D. Van Veen, "Partially adaptive beamformer design subject to worst case performance constraints," IEEE Trans. Signal Processing, vol. 42, no. 5, pp. 1218-1221, May 1994.

[13] J. F. Yang and M. Kaveh, "Digital realization of the real-time correlated signal-subspace transformation for array signal processing," in Proc. I988 ICASSP, 1988, vol. IV, pp. 2650-2653.

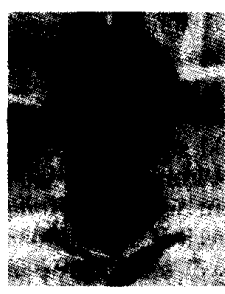

Feng Qian ( $S^{*} 90$ ) received the B.S. degree in electrical engineering from the University of Sciences and Technologies of China in 1985 . He received the M.A. in mathematics in 1988 and the Ph.D. in electrical engineering in 1993, both from the University of Wisconsin-Madison.

$\mathrm{He}$ is currently a Biomedical Engineer at the Datascope Corp. in New Jersey. His research interests are in the areas of array signal processing, adaptive signal processing algorithms, control system analysis and design, and numerical computation.

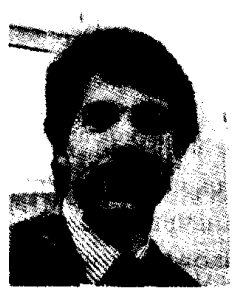

Barry D. Van Veen (S'81-M'86) was born in Green Bay, WI. He received the B.S. degree from Michigan Technological University in 1983 and the Ph.D. degree from the University of Colorado in 1986, both in electrical engineering. He was an ONR Fellow while working on the Ph.D. degree.

In the spring of 1987 he joined the Department of Electrical and Computer Engineering at the University of Colorado-Boulder. Since August, 1987, he has been with the Department of Electrical and Computer Engineering at the University of Wisconsin-Madison, where he is currently an Associate Professor. His research interests include signal processing for sensor arrays, spectrum estimation, adaptive filtering, and biomedical applications of signal processing.

Dr. Van Veen was a recipient of the 1989 Presidential Young Investigator Award and the 1990 IEEE Signal Processing Society Paper Award. 